\title{
Damaging fatigue cycles determination for random service loadings using mixed Weibull analysis
}

\author{
M. Mahmud ${ }^{1}$, S. Abdullah ${ }^{1,2}$, M.F.M. Yunoh ${ }^{1}$, A.K. Ariffin ${ }^{1,2}$ and Z.M. Nopiah ${ }^{1}$ \\ ${ }^{1}$ Department of Mechanical \& Materials Engineering, Faculty of Engineering \& Built \\ Environment, Universiti Kebangsaan Malaysia, \\ 43600 UKM Bangi, Selangor, Malaysia. \\ ${ }^{2}$ Centre for Automotive Research (CAR), Faculty of Engineering \& Built Environment, \\ 43600 UKM Bangi, Selangor, Malaysia. \\ *Email: shahrum@ukm.edu.my
}

\begin{abstract}
This paper investigates the fatigue damage behaviour of random service loading histories and suggests a criterion to determine fatigue damaging cycles by using probability values. The rainflow strain range distribution is often used in fatigue damage prediction. The existence of very small amplitude of cycles that do not significantly contribute to damage makes the prediction process unnecessarily complex. Therefore, a criterion to determine the size of damaging cycles that contribute significantly to total damage values is desired. In this study, two datasets were compared, i.e. the standard SAE road profile for the suspension system and an experimental data recorded using a strain gauge installed on a vehicle component. Fatigue damage was determined using the Coffin-Manson, Morrow and Smith-Watson-Topper models. The distribution of strain ranges was identified by distribution fitting process. The relation between strain range magnitude and $R M S$ values was explored using the probability of damage occurrence values. The results show that both sets of strain histories data fit well to a mixed Weibull distribution with $\mathrm{n}$ subpopulations. The probability of damage occurrence values obtained were very high, i.e. 0.9362 and 0.9987 , indicating very high chances of damage incurred by these damaging cycles. Therefore, $2 R M S$ values is suggested as a criterion for damaging cycles determination.
\end{abstract}

Keywords: damage; distribution; fatigue; loadings; mixed Weibull.

\section{INTRODUCTION}

Obtaining a highly accurate fatigue life prediction under service loadings with variable amplitude is still a challenging problem. One of the obstacles that is the nature of the time series signal of strains in service is often spectrum loadings, irregular and also nonstationary $[1,2]$. Another fundamental issue is in the extrapolation of complete design spectra from short time measured histories [3]. Therefore, enormous efforts have been exerted to improve life prediction and increase component durability and reliability by using random process methods so that the stochastic nature [4-6] of the fatigue process under service loading can be addressed properly. The multimodal probability density function used in the modelling of loading spectra of random processes was introduced by Nagode and Fajdiga [7]. This type of distribution is widely applied in reliability analysis of life data. For example, Attardi, Guida [8] applied a mixed Weibull regression model for the analysis of automotive warranty data because it involved two types of failure 
modes. In another study by Zhang and Xie [9], an upper truncated mixed Weibull distribution was explored to show its applicability for situations where the test data are bounded because of some restrictions. In the present paper, the similar idea of distribution truncation was applied for the determination of damaging fatigue cycles.

In reliability analysis, the distribution of strain range is used for damage prediction. In the context of fatigue damage, frequent low cycles can be meaningless [3]. From the design point of view, the most damaging cycles are more interesting to be observed rather than the frequent ones because they can be influenced by meaningless small amplitude vibrations and unfiltered noise. Hence, an analysis that can focus on the most relevant part of the amplitude distribution is desired. The problem is how to decide which cycles are worth including in the analysis so that the significant damage can be captured and the parameters are optimised. Therefore, this study aims to investigate the relations between strain range and root-mean-square $(R M S)$ values so that a suggestion for a criterion to determine the size of damaging cycles that contribute significantly to total damage values can be made. Another aspect concerning the fatigue damage distribution was also highlighted. In this context, a consistent description of the probability of damage occurrences is possible only if the damage distribution function is known. Therefore, two different sets of strain data from real components in service were analysed by strain-based fatigue analysis and statistical inferences to explore the resulting damage time series value, which is assumed to be a random variable. The mixed Weibull analysis was performed since the damage occurrences were expected to have a multimodal Weibull distribution.

\section{THEORETICAL BACKGROUND}

To describe the strain data, global statistics values were determined. The first statistic was the arithmetic mean. For $n$ number of observations, the mean was calculated as follows:

$$
\bar{x}=\frac{1}{n} \sum_{i=1}^{n} x_{i}
$$

The other commonly used statistic is the $R M S$. It represents the overall energy content of an oscillatory signal [10]. The equation is written as follows:

$$
R M S=\sqrt{\frac{1}{n} \sum_{i=1}^{n} x^{2}}
$$

To assess whether the data distribution was symmetrical or skewed, the third statistical moment was applied. The mathematical expression is given by:

$$
S=\frac{1}{n(R M S)^{3}} \sum_{i=1}^{n}\left(x_{i}-\bar{x}\right)^{3}
$$

The other statistic is the kurtosis value, which measures the peaked-ness of data distribution and it is highly sensitive to outliers. The formula is given by:

$$
K=\frac{1}{n(R M S)^{4}} \sum_{i=1}^{n}\left(x_{i}-\bar{x}\right)^{4}
$$

Three different models of strain-life were used to determine the number of cycles to failure, $N_{f}$. The Coffin-Manson (CM) model is normally used for loadings with zero mean stress effects. It was proposed after the works by Coffin in 1954 and Manson in 1965 [11]. The equation is as follows: 


$$
\varepsilon_{a}=\frac{\sigma_{f}^{\prime}}{E}\left(2 N_{f}\right)^{b}+\varepsilon_{f}^{\prime}\left(2 N_{f}\right)^{c}
$$

The inclusion of mean stress effects into life prediction makes the process more complex. Morrow suggested a model accounting for the mean stress effects in 1968. Morrow's mean stress method is given by

$$
\varepsilon_{a}=\frac{\sigma_{f}^{\prime}}{E}\left(1-\frac{\sigma_{m}}{\sigma_{f}^{\prime}}\right)\left(2 N_{f}\right)^{b}+\varepsilon_{f}^{\prime}\left(1-\frac{\sigma_{m}}{\sigma_{f}^{\prime}}\right)^{c / b}\left(2 N_{f}\right)^{c}
$$

where $\varepsilon_{a}$ is the total strain amplitude, $\sigma_{f}^{\prime}, b, \varepsilon_{f}^{\prime}$ and $c$ are considered to be material properties, $N_{f}$ is number of cycles to failure and $\sigma_{m}$ is the mean stress. Another model dealing with mean stress effects is the Smith-Watson-Topper model (often called SWT). It was suggested by Smith et al. in 1970 and the equation is:

$$
\sigma_{\max } \varepsilon_{a} E=\left(\sigma_{f}{ }^{\prime}\right)^{2}\left(2 N_{f}\right)^{2 b}+\sigma_{f}{ }^{\prime} \varepsilon_{f}{ }^{\prime} E\left(2 N_{f}\right)^{b+c}
$$

It is necessary to utilise a cumulative damage rule for the strain-based fatigue life prediction. The widely used Miner's rule was established deterministically as:

$$
D_{\text {TOT }}=\sum_{i} \frac{n_{i}}{N_{f i}}=1
$$

where $D_{\text {Tот }}$ is the total damage, $N_{f i}$ is the fatigue life of some materials according to the respective stress level and $n_{i}$ is the number of load cycles in fatigue test. The critical damage is assumed to be 1 . The damage per cycle can be calculated as:

$$
D=\frac{1}{N_{f i}}
$$

The distribution fitting procedure was performed by applying the Weibull analysis. Weibull distribution is often used to model life data for fatigue life prediction analysis. The mixed Weibull distribution was used to model the data that do not fall on a straight line on the Weibull probability plot [12]. The probability density function for a three-subpopulation distribution is written as:

$$
f_{1,2,3}(t)=\sum_{i=1}^{3} \frac{N_{i} \beta_{i}}{N \eta_{i}}\left(\frac{t}{\eta_{i}}\right)^{\beta_{i}-1} e^{-\left(\frac{t}{\eta_{i}}\right) \beta_{i}}
$$

where $N$ is the number of sample data, and $\beta$ and $\eta$ are distribution parameters.

\section{METHODS AND MATERIALS}

The first data set used in this study, $\mathrm{SAE}_{\text {Sus, }}$, was the typical strain history for a suspension system developed by the Society of Automotive Engineers (SAE). The detailed background to the data can be found in the work by Tucker and Stephen [13]. The other data set, $S_{\text {Exp }}$, was a set of experimental strain signals measured by strain gauge positioned on the coil spring component in a car suspension system driven in a rural area. The material used in the suspension was SAE 5160 and the properties are shown in Table 1. The diagrammatic process of the strain data collection is given in Figure 1. A data acquisition device and a computer were connected to the strain gauge for signal measurement by using a $500 \mathrm{~Hz}$ sampling rate frequency to ensure that all essential signals components were captured. The strain signals under service loadings consist of variable amplitude signals, as can be seen in Figure 2. The process flow of the methodology is shown in Figure 3. The first process to analyse the strain data was the 
computation of global statistics values. In order to identify and determine the number of cycles in each block of measured strain signals, the well-known rainflow counting method was performed. The rainflow counting method can be used to calculate the number of cycles in the irregular time histories. The resulting cycle counts were used to determine total damage endured by the component using the CM, Morrow and SWT models. Furthermore, the time series of damage evolution was obtained and damage occurrences across time and cycles were observed. Based on the damage occurrence time series, the distribution of the damage per cycle for $\mathrm{SAE}_{\mathrm{Sus}}$ and $\mathrm{S}_{\mathrm{Exp}}$ were investigated using the probability distribution.

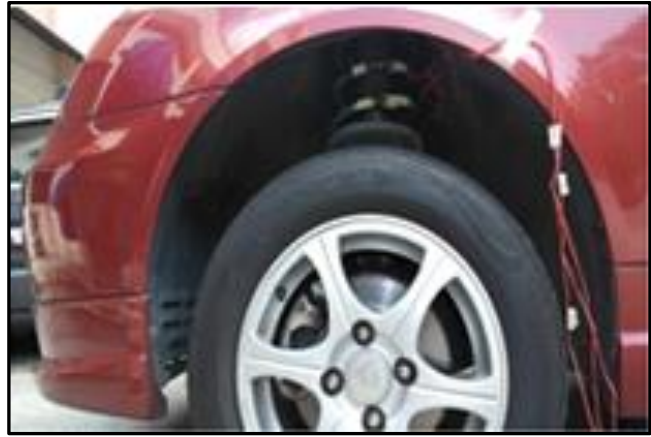

(a)

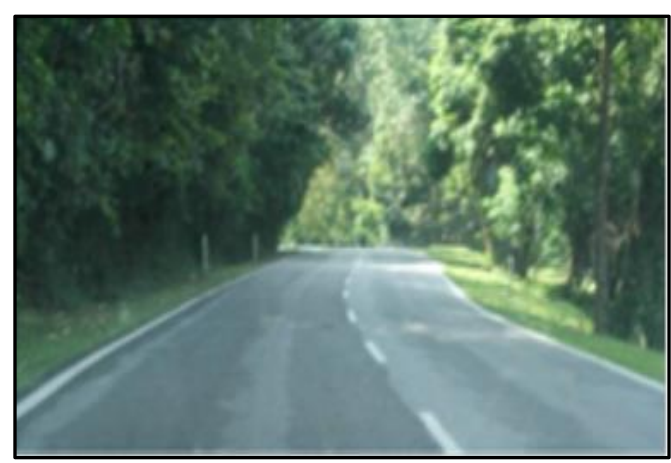

(c)

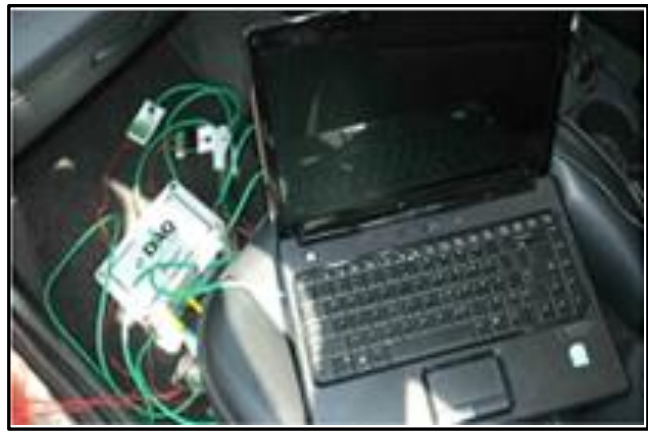

(b)

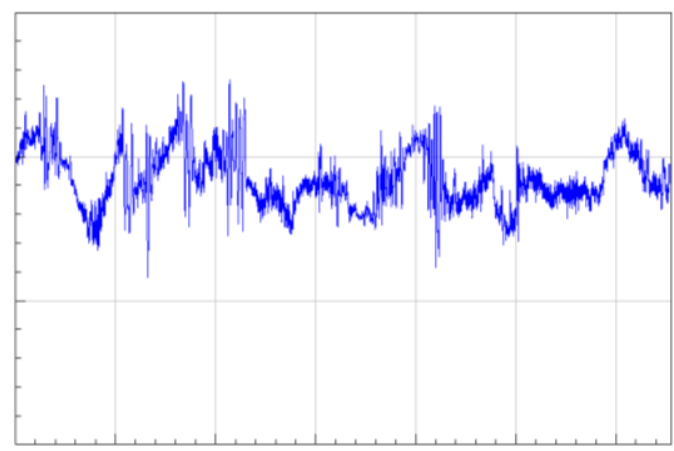

(d)

Figure 1. Diagrammatic process of the fatigue strain signal collection: (a) A strain gauge was attached to the vehicle coil spring surface. (b) The strain gauge was connected with a data acquisition device and computer. (c) The car was driven on the road. (d) Strain signals were obtained.

Table 1. Material properties of SAE 5160.

\begin{tabular}{ll}
\hline Yield strength (MPa) & 1487 \\
Ultimate tensile strength (MPa) & 1584 \\
Fatigue strength coefficient (MPa) & 2063 \\
Fatigue strength exponent & -0.0798 \\
Fatigue ductility exponent & -1.0498 \\
Fatigue ductility coefficient & 9.56 \\
\hline
\end{tabular}

A distribution fitting process was used to investigate the type of distribution characterised by the strain range values obtained from rainflow counting. Commercial mathematical software was used to determine the goodness-of-fit of the sample data to a pre-selected distribution. The computations of estimated parameters for the chosen 
distributions were performed using the maximum likelihood method. Finally, determination of damaging cycles was performed by using probability values. Previous studies conducted by Lambert [14] and Liou, $\mathrm{Wu}$ [15] suggested that most fatigue damage was caused by stress/strain values greater than 2 to 4 times the $R M S$ for a complete signal. Therefore, an $R M S$ value of 2 was used as the limit to define damaging cycles for analysis, i.e. cycles with strain range magnitude greater than $2 R M S$ were considered as 'damaging'.

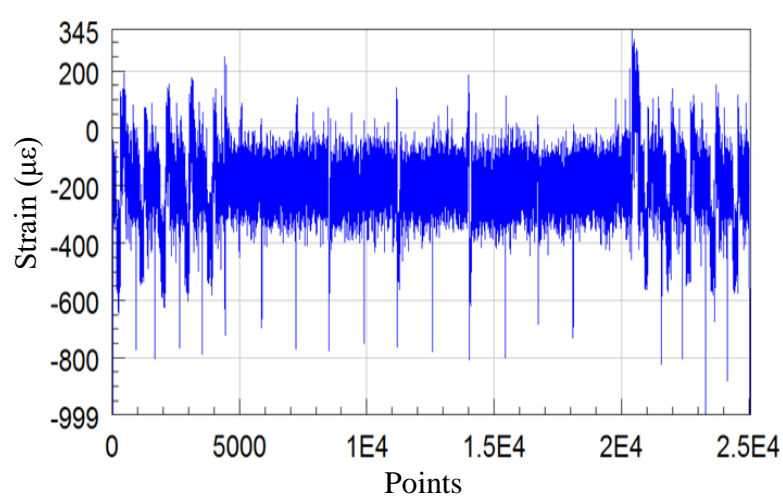

(a)

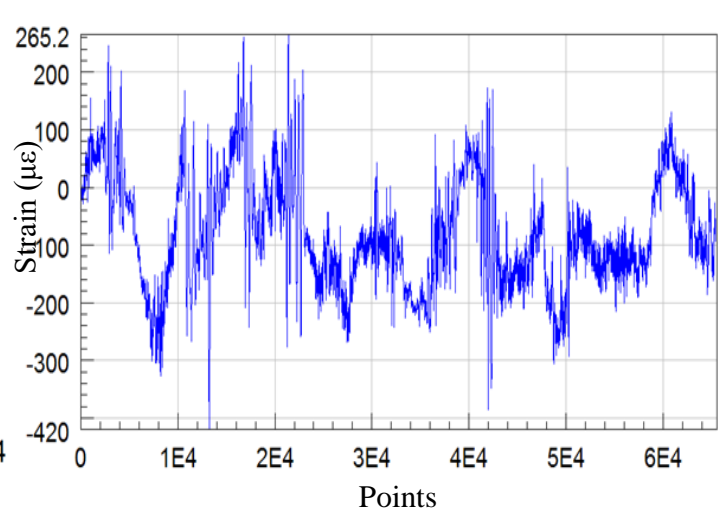

(b)

Figure 2. The time series plots of strain data for (a) SAESus and (b) $S_{\text {Exp }}$

\section{RESULTS AND DISCUSSION}

To describe the strain signals of $\mathrm{SAE}_{\text {Sus }}$ and $\mathrm{S}_{\mathrm{Exp}}$ statistically, the $R M S$ and kurtosis values were computed. Table 2 shows the summary statistics together with the fatigue analysis results, including the number of cycles counted and total damage per block. From the table, it is clear that $\mathrm{SAE}_{\text {Sus }}$ produced a higher value of $R M S$ compared to $\mathrm{S}_{\mathrm{Exp}}$ by $95 \%$. This finding is owing to the fact that $\mathrm{SAE}_{\text {Sus }}$ have more signals with high amplitudes which result in more oscillatory energy [16]. Because $S_{\text {Exp }}$ data was recorded from a car driven on rural road where mostly braking and cornering manoeuvres are involved, therefore the signal would have mean stress effects. The SAE $E_{\text {Sus }}$ also obviously have compressive mean stress effects that occurred across the signal series. Based on the kurtosis values, both signals are not Gaussian and are also non-stationary. For a Gaussian process, the kurtosis value is approximately 3.0. A kurtosis value of 4.32 in SAEsus indicates the existence of numerous extreme values in the data set. The total damage value for $S_{E x p}$ is lower compared to $\mathrm{SAE}_{\text {Sus. }}$ This result is consistent with $\mathrm{S}_{\mathrm{Exp}}$ having a lower value of $R M S$. From the fatigue damage values in Table 2, it can be seen that even though the values are very close to each other, there are still differences. Nevertheless, the SWT model has been shown to correlate mean stress better for a wider range of materials and is therefore more promising for general use [17]. Consistently, the expected component lives under both loading data listed in the last column shows that $S_{\operatorname{Exp}}$ has a higher expected life for all three models than that for $\mathrm{SAE}_{\text {Sus. }}$.

Figure 4 shows the distribution of rainflow cycles across the strain ranges and the mean value for both signals. From the rainflow cycle counting, the list of maximum and minimum values of each cycle was obtained. The strain range for each cycle was calculated by subtracting each minimum value from the maximum. The result for $\mathrm{SAE}_{\text {Sus }}$ demonstrates that all cycles have a strain range greater than zero. Theoretically, these high strain ranges will give more damaging effects compared to the low strain range [18]. Other than that, the distributions of the cycles are asymmetric, highlighting the non- 
Gaussian behaviour of the signal. For $S_{\text {Exp }}$, apparently there are more cycles with low

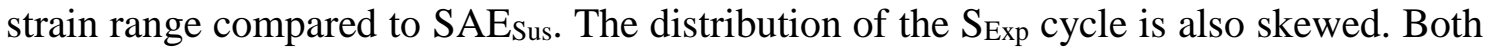
signals have cycles with a very high strain range and, theoretically, these cycles give more damaging effects compared to the low strain range.

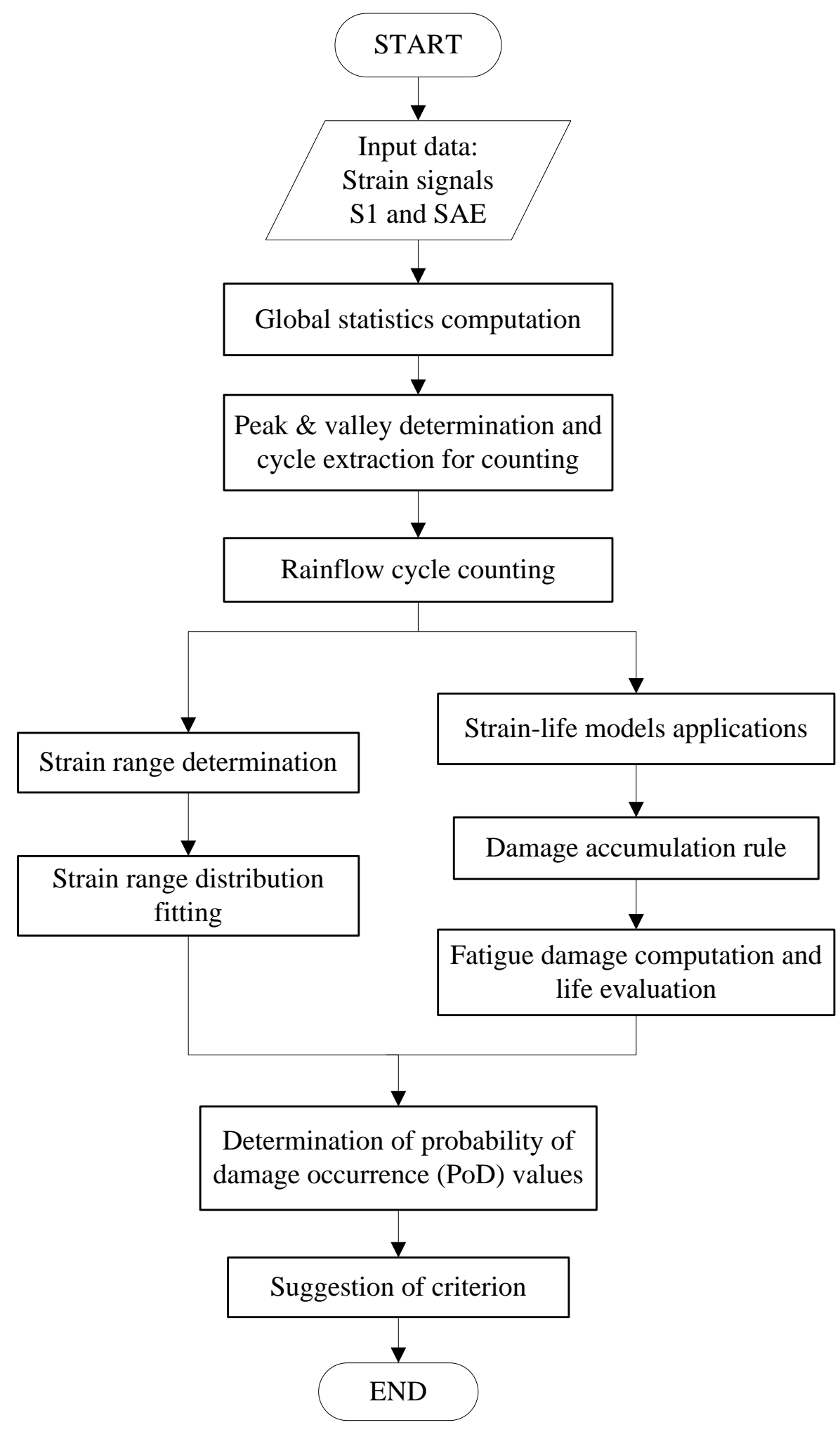

Figure 3. The process flow of the methodology. 

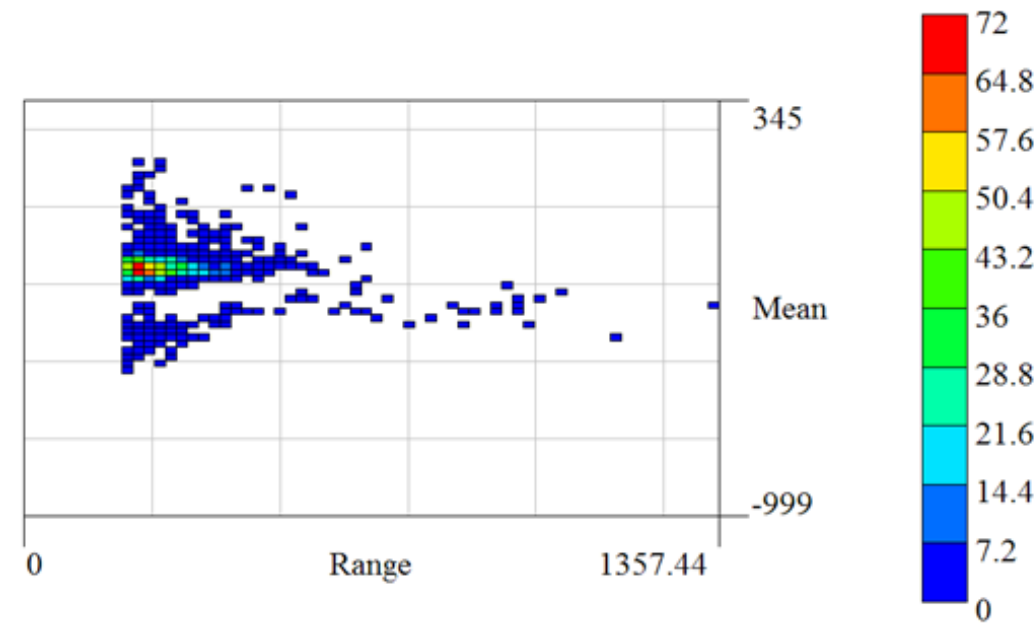

(a)

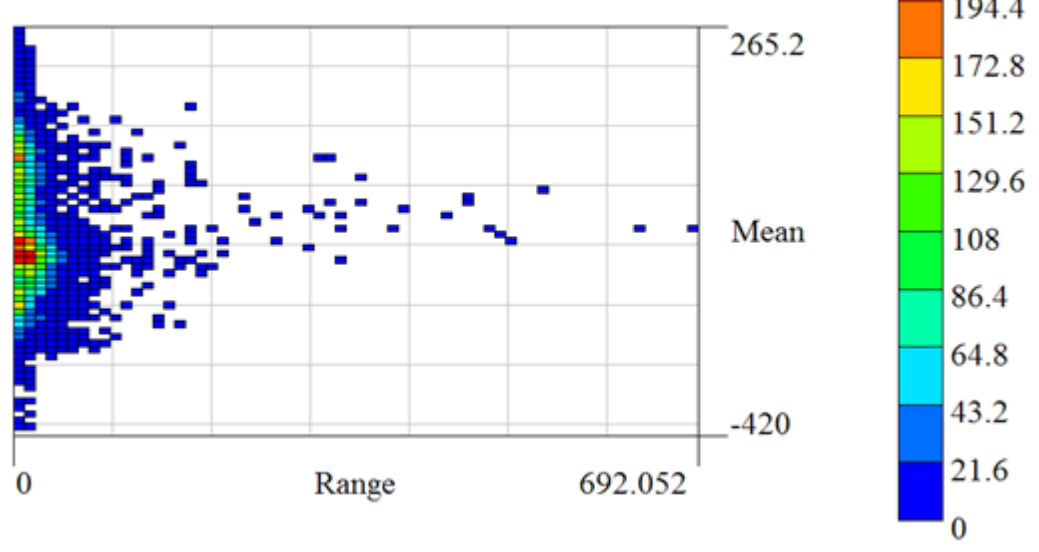

(b)

Figure 4. The cycle-counted distribution for (a) SAESus and (b) $S_{\text {Exp }}$.

Table 2. Summary statistics and fatigue analysis results of strain signals.

\begin{tabular}{|c|c|c|c|c|c|c|c|c|}
\hline Data & $\begin{array}{c}\mathrm{N} \\
\text { (cycles) }\end{array}$ & $\begin{array}{c}\text { Mean } \\
(\mu \varepsilon)\end{array}$ & Skewness & $\begin{array}{l}R M S \\
(\mu \varepsilon)\end{array}$ & Kurtosis & \multicolumn{2}{|c|}{$\begin{array}{l}\text { Fatigue Damage } \\
\text { (damage/block) }\end{array}$} & $\begin{array}{c}\text { Expected life } \\
\text { (repeated } \\
\text { blocks) }\end{array}$ \\
\hline $\mathrm{SAE}_{\text {Sus }}$ & 1253 & -206.6 & -0.37 & 246.6 & 4.32 & $\begin{array}{c}\text { CF } \\
\text { Morrow } \\
\text { SWT }\end{array}$ & $\begin{array}{l}1.70 \times 10^{-3} \\
1.70 \times 10^{-3} \\
1.64 \times 10^{-3}\end{array}$ & $\begin{array}{l}590.1 \\
590.1 \\
609.4\end{array}$ \\
\hline$S_{\text {Exp }}$ & 9768 & -78.9 & 0.36 & 126.4 & 2.82 & $\begin{array}{l}\mathrm{CF} \\
\text { Morrow } \\
\text { SWT }\end{array}$ & $\begin{array}{l}5.15 \times 10^{-4} \\
5.03 \times 10^{-4} \\
4.89 \times 10^{-4}\end{array}$ & $\begin{array}{l}1942.2 \\
1988.8 \\
2045.4\end{array}$ \\
\hline
\end{tabular}

The histograms to demonstrate the distribution of damage across the range values are given in Figure 5. Apparently, a higher strain range will cause higher fatigue damage. There were more damaging cycles in $\mathrm{SAE}_{\text {Sus }}$ compared to $\mathrm{S}_{\mathrm{Exp}}$. The results of the fatigue damage values as shown previously in Table 1 are very close to each other. Nevertheless, 
the Morrow model has been suggested to provide better life prediction for signals comprised mostly of compression data $[19,20]$. In general, $\mathrm{SAE}_{\text {Sus }}$ gives the highest damage compared to $\mathrm{S}_{\text {Exp. }}$. This outcome is consistent with the $R M S$ results, which measure the oscillatory energy content of the signals, i.e. the higher the energy, the more damaging it is in terms of fatigue.

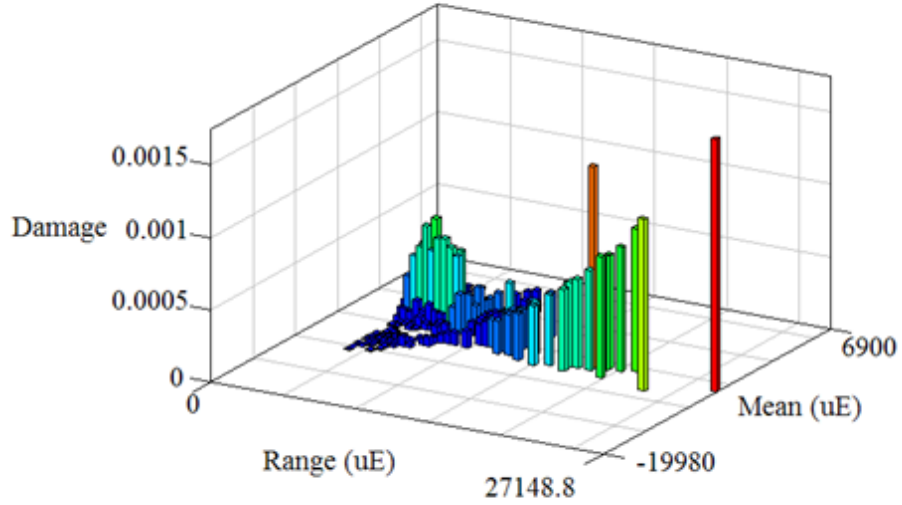

(a)

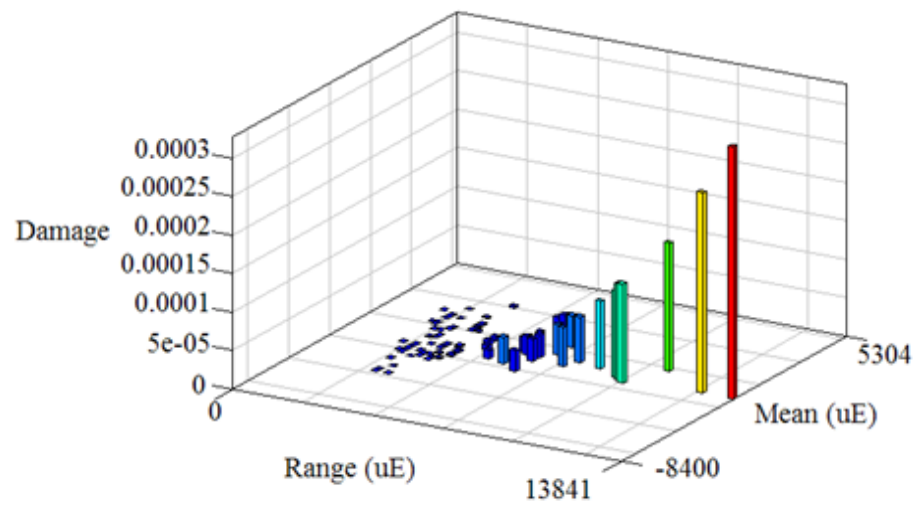

(b)
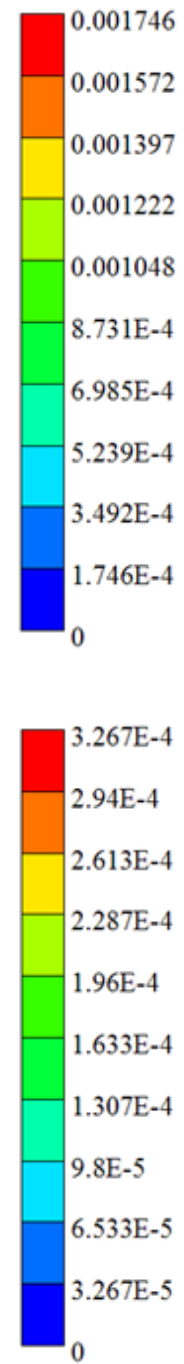

Figure 5. The damage histograms for (a) $\mathrm{SAE}_{\text {Sus }}$ and (b) $\mathrm{S}_{\text {Exp. }}$.

The strain range can be considered as the size of the respective cycle. The higher the strain range value, the larger the fatigue damage. Therefore, it is interesting to see the statistical distribution and scatter of the strain range for both signals. Figures 6 (a) and (b) show the probability plots for mixed Weibull with two subpopulations. By applying a series of distribution fitting processes, it was found that the most suitable distribution for the datasets was the mixed Weibull distribution. The corresponding parameter values are shown in Table 3. The probability plots shown in Figure 6 demonstrate that there are various slopes in each plot. This indicates the existence of multiple peaks in the distribution of data. That is why the mixed Weibull is an excellent choice of distribution for modelling the data behaviour. Furthermore, the plots show that the fitting for higher 
values of strain range is better than the lower values. However, this study emphasises the damaging part more, which is the higher magnitude of strain range rather than the small amplitude of cycles.

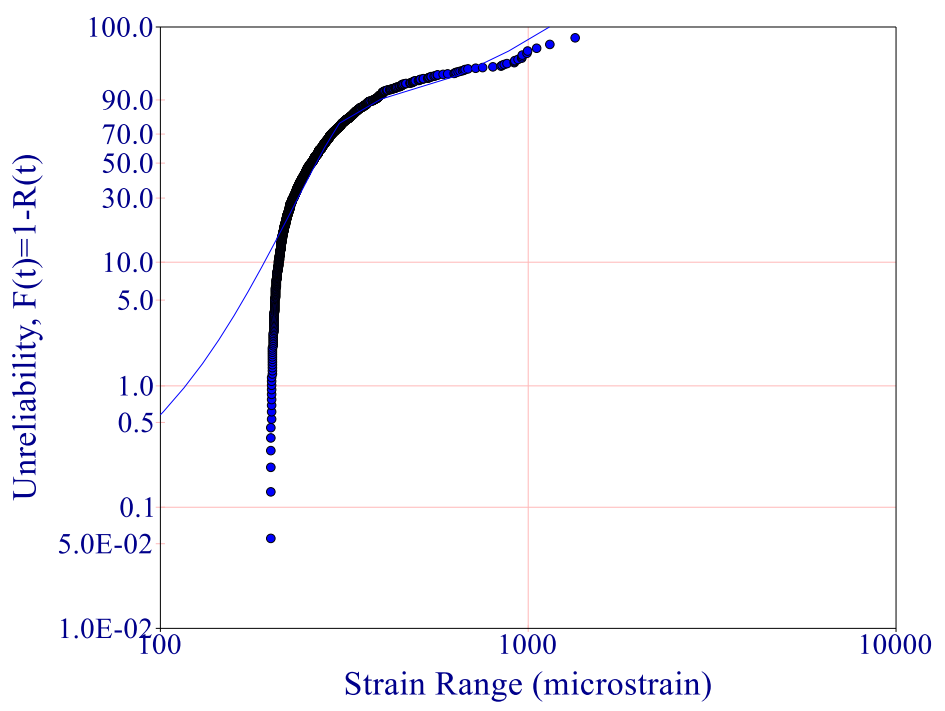

(a)

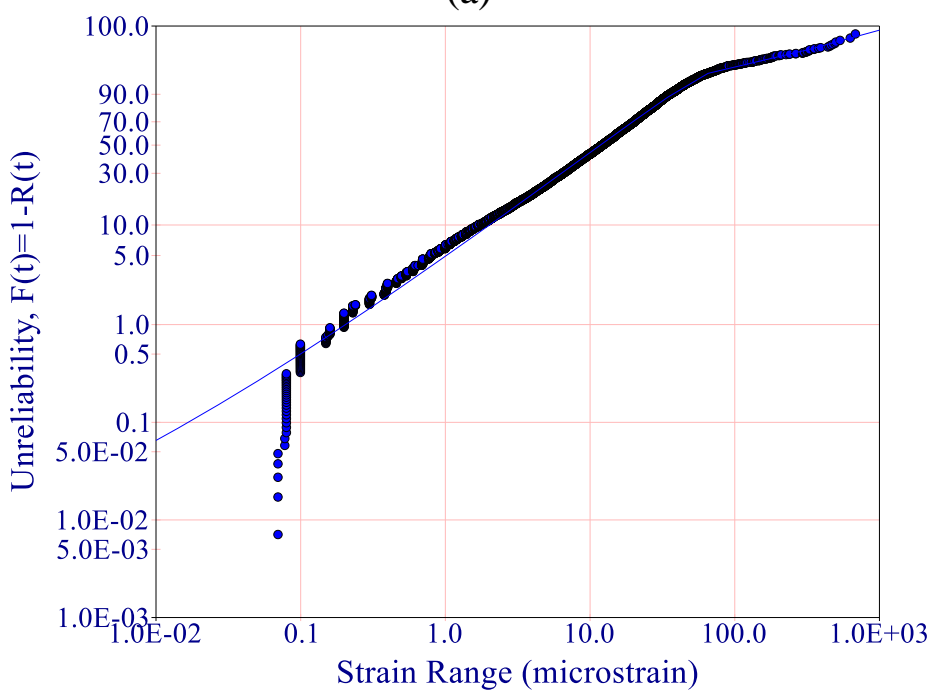

(b)

Figure 6. The probability plots of (a) $\mathrm{SAE}_{\text {Sus }}$ and (b) $\mathrm{S}_{\text {Exp. }}$

Table 3. Statistical distribution and parameters for strain range.

\begin{tabular}{llcccc}
\hline Data & Distribution type & Subpopulation & $\beta$ & $\eta$ & Portion \\
\hline Range of & Mixed Weibull (2-subpop) & I & 6.42 & 273 & 0.82 \\
SAE & & II & 2.34 & 483 & 0.18 \\
Range of & Mixed Weibull (2-subpop) & I & 1.10 & 16 & 0.94 \\
S $_{\text {Exp }}$ & & II & 0.61 & 37 & 0.06 \\
\hline
\end{tabular}

\section{Fatigue Damage Distribution}

The schematic diagram of fatigue damage values endured by a component across a cycle series can be observed in Figure 7 (a). Theoretically, high values of strain amplitudes are 
associated with high damage values. It is interesting to know the distribution of damage for both strain data sets so that one can see the type of distribution that characterises the fatigue damage in the samples. A schematic diagram of the statistical data distribution for fatigue damage occurrences is shown in Figure 7 (b).

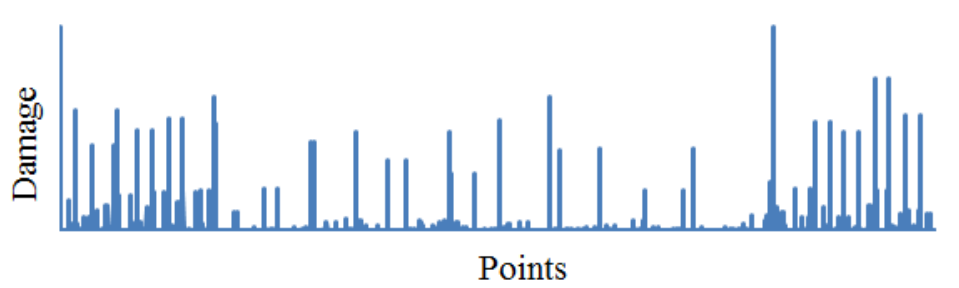

(a)

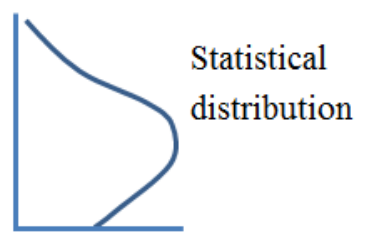

(b)

Figure 7. Schematic diagram of damage data series distribution fitting: (a) The damage value series and (b) the respective statistical distribution.

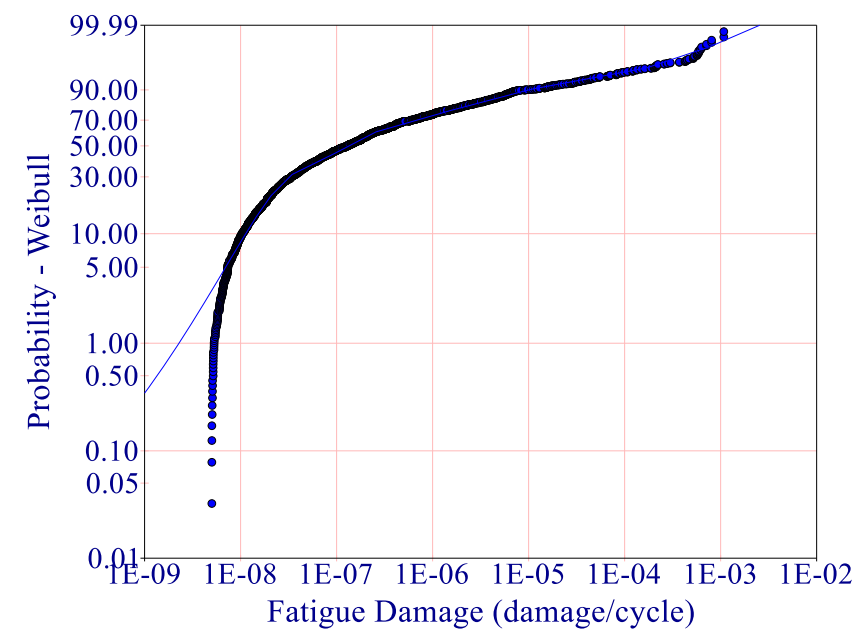

(a)

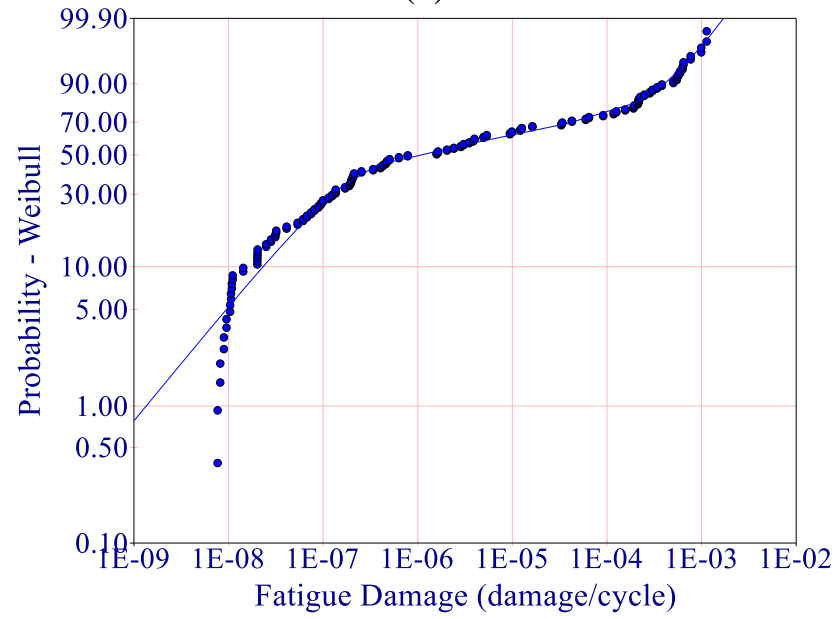

(b)

Figure 8 . The m-subpopulation-mixed Weibull probability plots of damage per cycle distribution for (a) SAESus and (b) $\mathrm{S}_{\text {Exp. }}$.

Figure 8 shows the best-fitted probability plots for both fatigue damage series. Specifically, the results show that the damage data has a mixed Weibull distribution with three or four subpopulation types. Table 4 details the estimated parameter values. The 
shape (or slope) of the distribution curve is determined by $\beta$ while $\eta$ is the scale parameter in the damage unit. The portions are shown accordingly in the last column of Table 4 and are indicative of the division of total data into subpopulations. The number of subpopulations is according to the number of multimodal characteristics of the data. The mixed Weibull analysis was chosen to model the data because the damage data has an Sshape when plotted on the probability paper. This indicates a multimodal type of data requiring individual mathematical treatment. This type of data also can be found in life data reliability analyses when more than one failure mode exists. In the context of fatigue damage behaviour, apparently there are several groups of different damage values resulting from different clusters of amplitudes in the loading spectrum.

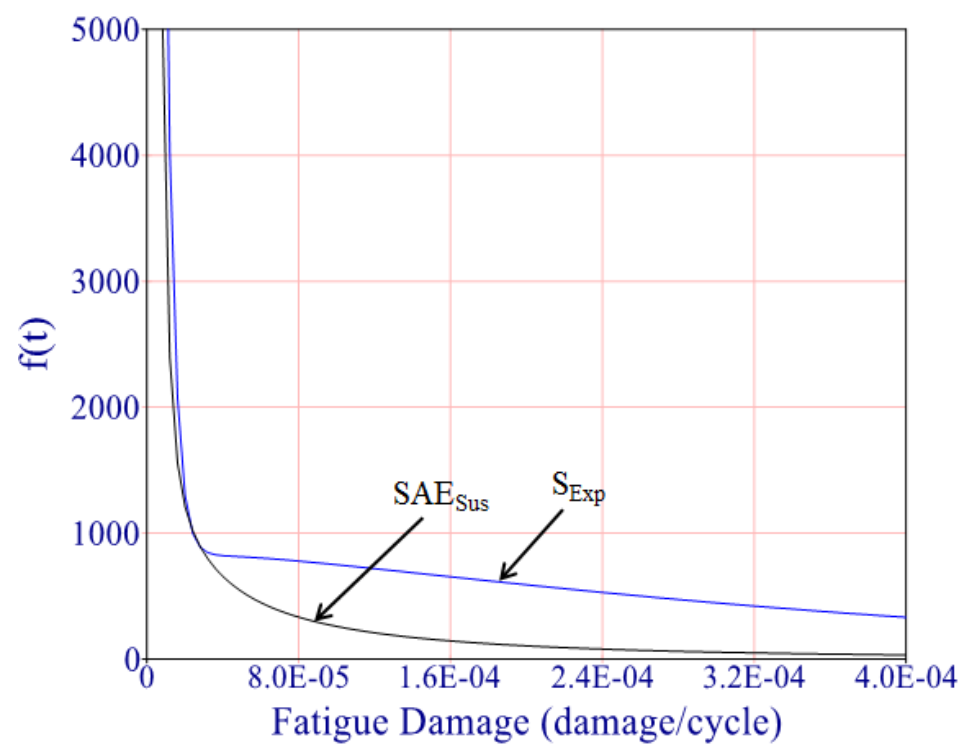

(a)

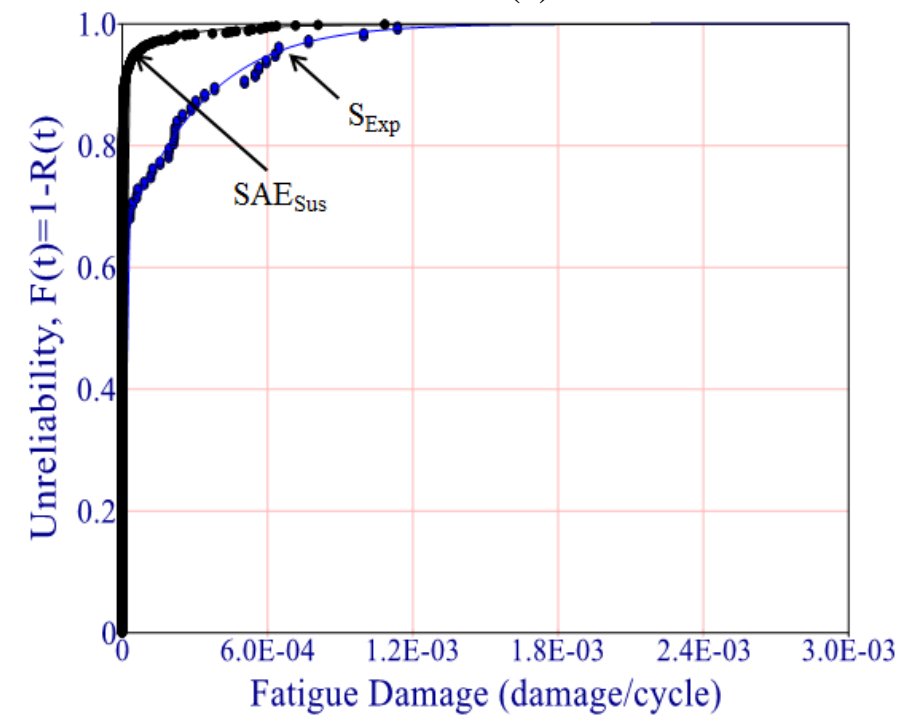

(b)

Figure 9. The overlay distribution plots for both signals: (a) the probability density function and (b) the cumulative distribution function.

Figure 9(a) shows the overlay plot of the PDF for both signals. It is seen that the tail of the $\mathrm{S}_{\text {Exp }}$ distribution is thicker than that for $\mathrm{SAE}_{\text {Sus. }}$ This is owing to $\mathrm{S}_{\operatorname{Exp}}$ having more low strain signals, which cause low damage values. Hence, there are more 
occurrences of low damage values on the tail of $S_{\text {Exp. }}$ The respective overlay cumulative distribution function (CDF) plot is shown in Figure 9(b). The CDF indicates that the damage of $\mathrm{SAE}_{\text {Sus }}$ more rapidly increases in cumulative probability values compared to

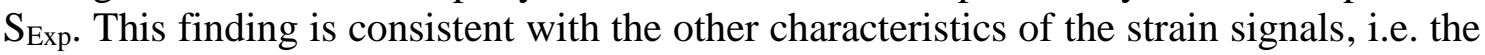
$\mathrm{SAE}_{\text {Sus }}$ have higher amplitudes and oscillatory energy. This means that SAESus also have a higher probability of damage occurrences. Therefore, the overlay CDF in Figure 9(b) demonstrates that the increment in probability of damage occurrences of SAESus is higher compared to that for $\mathrm{S}_{\mathrm{Exp}}$.

Table 4. Statistical distribution and parameters for fatigue damage.

\begin{tabular}{lccccc}
\hline Data & Distribution type & $\begin{array}{c}\text { Sub- } \\
\text { population }\end{array}$ & $\beta$ & $\eta$ & Portion \\
\hline Damage & Mixed Weibull (4-subpop) & I & 1.81 & $2.03 \times 10^{-8}$ & 0.25 \\
series of & & II & 1.10 & $1.45 \times 10^{-7}$ & 0.36 \\
SAESus & & III & 0.83 & $2.00 \times 10^{-6}$ & 0.25 \\
& & IV & 0.53 & $6.20 \times 10^{-5}$ & 0.14 \\
Damage & Mixed Weibull (3-subpop) & I & 0.84 & $1.27 \times 10^{-7}$ & 0.47 \\
series of & & II & 1.08 & $5.00 \times 10^{-6}$ & 0.19 \\
SExp & & III & 1.09 & $3.38 \times 10^{-4}$ & 0.34 \\
\hline
\end{tabular}

\section{Damaging Cycles Determination}

The findings about strain ranges distribution are useful to make inferences using probabilistic interpretations. The proposed criterion was based on the PoD values. The PoD can be derived directly according to a specified value of strain range from the CDF. The specified value in this case is the $2 R M S$ based on previous research by Lambert [14] and Liou, Wu [15]. A schematic diagram of the PoD determination is shown in Figure 10. A specific PoD value can be obtained by determining the probability based on the strain range of $2 R M S$. For random loading data considered in this study, the corresponding PoD values are tabulated in Table 5. The values are 0.9362 and 0.9987 , indicating very high chances of damage being incurred by these damaging cycles. Therefore, it is suggested that $2 R M S$ values as a criterion for damaging cycles determination for reliability analysis.

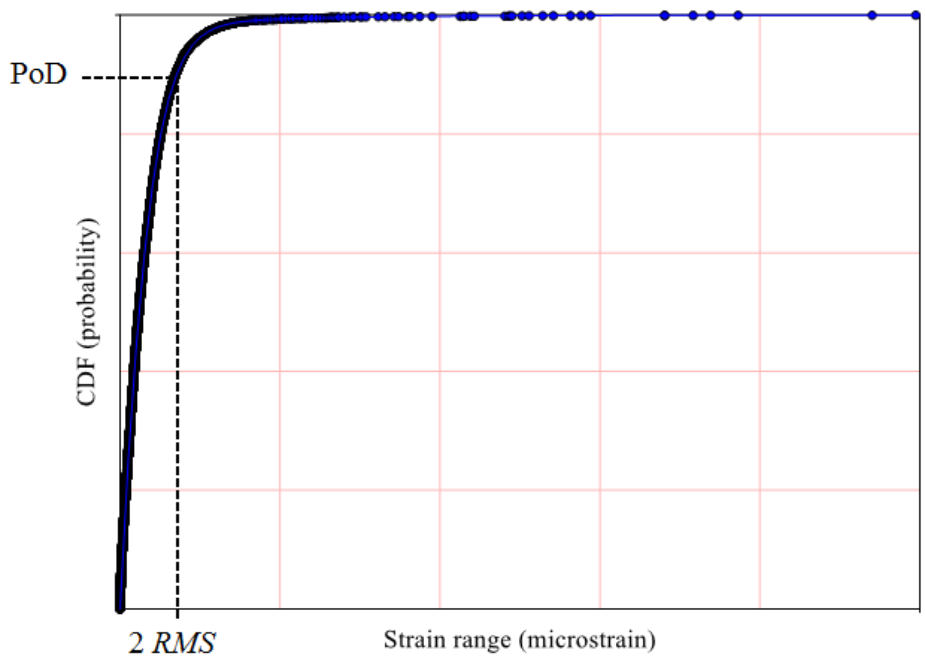


Figure 10. Schematic of CDF plot for PoD determination.

Table 5. Probability of damage occurrence for cycles range greater than $2 R M S$.

\begin{tabular}{ccc}
\hline Signal & $\mathbf{2} \boldsymbol{R M S}(\boldsymbol{\mu \varepsilon})$ & $\begin{array}{c}\text { Probability } \\
(\mathbf{P o D})\end{array}$ \\
\hline $\mathrm{S}_{\text {Exp }}$ & 140 & 0.9987 \\
$\mathrm{SAE}_{\mathrm{Sus}}$ & 493 & 0.9362 \\
\hline
\end{tabular}

\section{CONCLUSIONS}

Two strain signals data sets in service were analysed and compared. The fatigue damage series distribution of these data has been modelled successfully by a mixed Weibull distribution. A criterion for determining the level of damaging cycles for the reliability analysis has been explored in this study. Six strain signal data sets obtained from experiments were used for the analysis. Fatigue damage was determined using the CM, Morrow and SWT models. The emphasis of this work is on the relation of strain range distribution and the $R M S$ values so that the damaging part of the strain range distribution can be identified. The probabilities of damage occurrence values were also very high indicating very high chances of damage being incurred by these damaging cycles. Therefore, it is suggested here that the criterion for damaging cycles determination for reliability analysis is the cycles with strain ranges greater than $2 R M S$. By focusing on the damaging cycles, the distribution parameters are optimised and the time for analysis is shortened. Another important conclusion is that the fatigue damage series values fit well to a mixed Weibull distribution even though both signals have the distinct nature of time series data, e.g. the kurtosis and $R M S$. Based on the distribution, inferences about the behaviour of fatigue strain loading in service can be stated using probability values. The fatigue damage of a component in a suspension system can also be characterised by a multimodal distribution, similar to the case of loading spectra as in previous studies.

\section{ACKNOWLEDGEMENTS}

The authors wish to express their gratitude to Ministry of Higher Education Malaysia via Universiti Kebangsaan Malaysia for supporting this research project

\section{REFERENCES}

[1] Mohamed SAN, Abdullah S, Arifin A, Ariffin AK, Padzi MM. Characterization of the biaxial fatigue behaviour on medium carbon steel using the strain-life approach. International Journal of Automotive and Mechanical Engineering. 2016;13:3262-77.

[2] Patel CB, Gohil PP, Borhade B. Modeling and Vibration Analysis of Road Profile Measuring System. International Journal of Automotive and Mechanical Engineering. 2010;1:13-28.

[3] Tovo R. A damage-based evaluation of probability density distribution for rainflow ranges from random processes. International Journal of Fatigue. 2000;22:425-9.

[4] Liu Y, Mahadevan S. Stochastic fatigue damage modeling under variable amplitude loading. International Journal of Fatigue. 2007;29:1149-61. 
[5] Shen $\mathrm{H}$, Lin J, Mu E. Probabilistic model on stochastic fatigue damage. International Journal of Fatigue. 2000;22:569-72.

[6] Echard B, Gayton N, Bignonnet A. A reliability analysis method for fatigue design. International Journal of Fatigue. 2014;59:292-300.

[7] Nagode M, Fajdiga M. A general multi-modal probability density function suitable for the rainflow ranges of stationary random processes. International Journal of Fatigue. 1998;20:211-23.

[8] Attardi L, Guida M, Pulcini G. A mixed-Weibull regression model for the analysis of automotive warranty data. Reliability Engineering \& System Safety. 2005;87:265-73.

[9] Zhang T, Xie M. On the upper truncated Weibull distribution and its reliability implications. Reliability Engineering \& System Safety. 2011;96:194-200.

[10] Abdullah S, Choi J, Giacomin J, Yates J. Bump extraction algorithm for variable amplitude fatigue loading. International Journal of Fatigue. 2006;28:675-91.

[11] Dowling NE. Mechanical Behavior of Materials. 4th ed: Pearson Education Limited; 2013.

[12] Klemenc J, Fajdiga M. Improved modelling of the loading spectra using a mixture model approach. International Journal of Fatigue. 2008;30:1298-313.

[13] Tucker L, Stephen B. The SAE cumulative fatigue damage test program. In: Wetzel RM: Fatigue under complex loading. 1975;SAE:3-53.

[14] Lambert RG. Plastic work interaction damage rule applied to narrow-band Gaussian random stress situations. Journal of Pressure Vessel Technology. 1998;110:88-90.

[15] Liou HY, Wu WF, Shin CS. A modified model for the estimation of fatigue life derived from random vibration theory. Probabilistic Engineering Mechanics. 1999;14:281-8.

[16] Abdullah S. Wavelet bump extraction (WBE) for editing variable amplitude fatigue loadings. United Kingdom: The University of Sheffield; 2005.

[17] Stephens RI, Fatemi A, Stephens RR, Fuchs HO. Metal Fatigue in Engineering. 2 ed. Canada: John Wiley \& Sons, Inc; 2001.

[18] Schijve J. Fatigue of Structures and Materials. 2nd ed: Springer; 2009.

[19] Halford GR, Gallagher JP. Fatigue and Fracture Mechanics: 31st Volume: American Society for Testing and Materials; 2000.

[20] Ince A, Glinka G. A modification of Morrow and Smith-Watson-Topper mean stress correction models. Fatigue \& Fracture of Engineering Materials \& Structures. 2011;34:854-67. 